PAPER

\title{
Early computed tomography features in extensive middle cerebral artery territory infarct: prediction of survival
}

\author{
W W M Lam, T W H Leung, W C W Chu, D T K Yeung, L K S Wong, W S Poon
}

See end of article for authors' affiliations

Correspondence to: W W M Lam, Department of Diagnostic Radiology and Organ Imaging The Chinese University of Hong Kong, c/o Rm 27029, X-ray Department, Prince of Wales Hospital, Ngan Shing Street, Shatin, N.T., Hong Kong; wynnie@cuhk.edu.hk

Received

29 December 2003

In revised form

23 June 2004

Accepted 24 June 2004
J Neurol Neurosurg Psychiatry 2005;76:354-357. doi: 10.1136/jnnp.2003.035055

Background: To assess the predictive value of prognosis of different computed tomography (CT) features and National Institutes of Health Stroke Scale score (NIHSS) in acute extensive middle cerebral artery (MCA) infarct.

Methods: Fifty five patients with acute extensive MCA infarct had the CT performed within 24 hours of the onset of symptoms. A total of 11 CT features were analysed. The age distribution, presence of risk factors, presence of individual CT feature, the total CT score, and the NIHSS were correlated with the 30 day mortality.

Results: Single explanatory variable analysis showed NIHSS, presence of midline shift, midline shift of more than $1 \mathrm{~cm}$, extent of infarct, presence of hydrocephalus, effacement of subarachnoid space/cella media, attenuation of corticomedullary differentiation, and total CT score were associated with the 30 day mortality. Both extent of infarct $>67 \%$ and attenuation of corticomedullary differentiation gave a sensitivity and specificity of $93 \%$ and $95 \%$, respectively, for the prediction of survival. Logistic regression analysis showed that the extent of infarct and NIHSS were the only independent predictors.

Conclusions: CT features and admission NIHSS are important parameters for prediction of survival in extensive MCA infarct.
$\mathrm{E}$ xtensive middle cerebral artery (MCA) infarct is relatively uncommon. It accounts for $3.25 \%$ of all acute stroke patients in our centre (which represents a major regional hospital). Despite its relatively low incidence, it has significant clinical impact. Space occupying effect is more likely to be demonstrated in extensive infarct and the mortality is higher. It is therefore essential to identify patients who are likely to do badly and might benefit from interventions such as decompressive craniectomy. In most centres, computed tomography (CT) is often the first and the only imaging for evaluation of these patients. We are therefore assessing the prognostic value of individual radiological sign demonstrated by CT performed within 24 hours of the clinical onset of signs and symptoms.

\section{METHODS}

The data file of all acute stroke adult patients admitted to our stroke unit from January 2000 to January 2002 was retrospectively evaluated. A total of 2183 acute stroke patients were admitted within this time interval. Out of these patients, a total of 71 patients had extensive MCA infarct finally diagnosed. The diagnosis was established when either a bilobar (frontoparietal or parieto-occipital) or a hemispheric infarct was demonstrated by the initial or follow up CT examination. Only 55 of these patients ( 31 female, 24 male, age range from 53-93 years, with a mean of 75.8 years) had the CT performed in the first 24 hours after onset of clinical symptoms. The other 16 patients had CT examinations performed after 24 hours because of late presentation to our hospital. All examinations were performed with the HiSpeed Advantage (GE Medical Systems, Milwaukee, USA). All these 55 patients presented with total anterior circulation stroke clinically. The skull base to the posterior cranial fossa was scanned with $5 \mathrm{~mm}$ axial cuts and the supratentorial region to the vertex was scanned with $10 \mathrm{~mm}$ axial consecutive slices. All CT images of these 55 patients were retrieved in the workstation (Advantage Window 3.1, Milwaukee, USA) for analysis. All the CT images were analysed independently by two fully qualified radiologists (WWML, WCWC) who were informed of the diagnosis of extensive MCA infarction but blinded to the patients' outcomes. The images were then scored and graded by the diagnostic criteria below. Any discrepancy in interpretation of films and scoring of criteria were resolved by the third radiologist (DTKY).

Clinically, all these patients had been examined by a qualified neurologist and National Institutes of Health Stroke Scale score (NIHSS) on admission was recorded. The presence or absence of atrial fibrillation, diabetes mellitus, hypertension, hyperlipidaemia, ischaemic heart disease, and smoking were all recorded in the medical diary.

All of these patients were managed conservatively and none of them had decompressive craniectomy. The 30 day mortality of the patients was recorded.

\section{Interpretation of CT images}

All initial CT examinations were scored according to the following diagnostic criteria:

- Presence of midline shift: it was scored as 0 when midline shift was absent. A score of 1 was given when midline shift was present. When midline shift was present, the extent of midline shift was measured by electronic caliper on the console. An additional score of 1 was given when the midline shift was more than $1 \mathrm{~cm}$.

- The extent of MCA infarct: score 0 was given when there was $<33 \%$ of involvement of the MCA territory. Score l was given when there was $>33 \%$ but $<67 \%$ of involvement, score 2 was given when there was $>67 \%$ involvement.

- The presence of concomitant acute infarct in other territories: a score of 0 was given when there was no

Abbreviations: $\mathrm{CT}$, computed tomography; MCA, middle cerebral artery; NIHSS, National Institutes of Health Stroke Scale score 
concomitant acute infarct. A score of 1 was given when there was concomitant acute infarct in contralateral hemisphere. A score of 2 was given when there was concomitant acute infarct in ipsilateral anterior cerebral or posterior cerebral artery with or without acute infarct in contralateral hemisphere.

- Presence of obstructive hydrocephalus: a score 0 was given for absence of hydrocephalus and a score 1 was given when obstructive hydrocephalus was detected.

- Presence of tentorial herniation: a score 0 was given when there was absence of the sign. A score 1 was given when there was presence of sign.

- Presence of haemorrhagic foci: a score 0 was given when there was no haemorrhagic foci demonstrated on the acute infarcted area. A score 1 was given when there was haemorrhagic foci.

- Effacement of quadrigeminal, ambient, and interpeduncular cistern: a score 0 was given when the basal cisterns were not effaced. Score 1 was given when there was effacement of any of these cisterns.

- Presence of hyperdense MCA sign (fig 1): score 0 given when the sign was absent, score 1 was given when the sign was present.

- Effacement of subarachnoid space and cella media: score 0 was given when there was no significant effacement and a score 1 was given when the cella media was compressed or when subarachnoid space of the whole hemisphere was effaced.

- Attenuation of corticomedullary contrast (fig 2): score 0 was given when there was no significant attenuation of the corticomedullary differentiation. Score 1 was given when there was attenuation of the corticomedullary differentiation throughout the entire MCA territory.

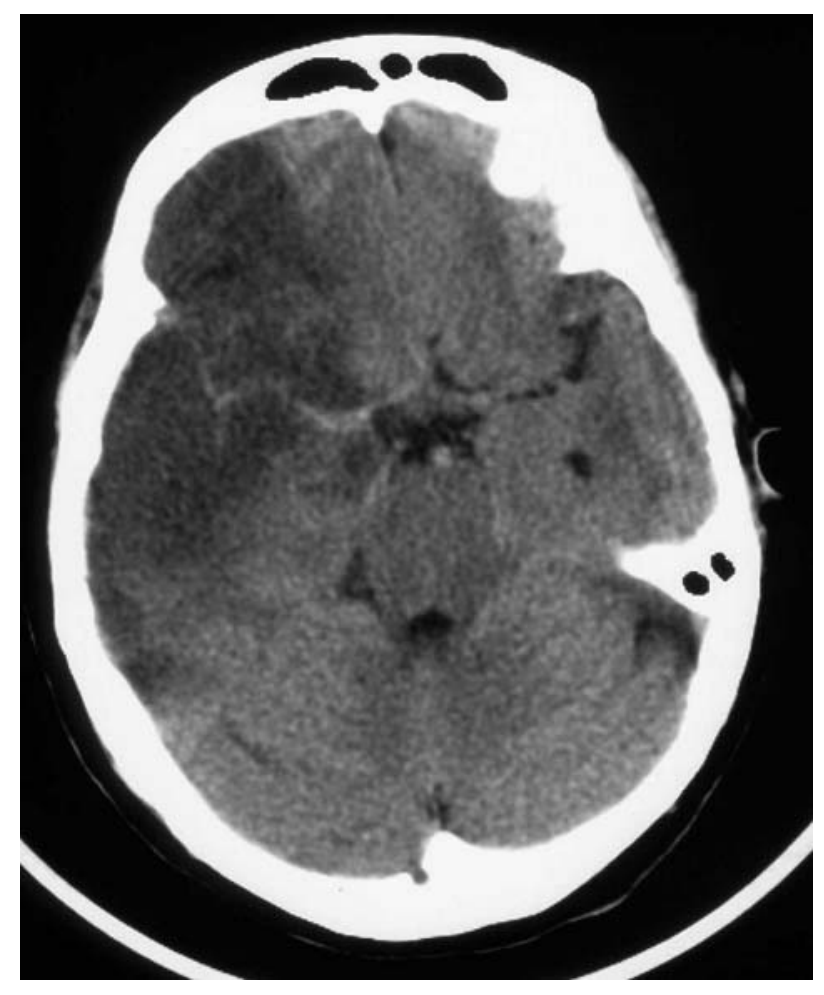

Figure 1 Computed tomography brain scan showing hyperdense right middle cerebral artery.

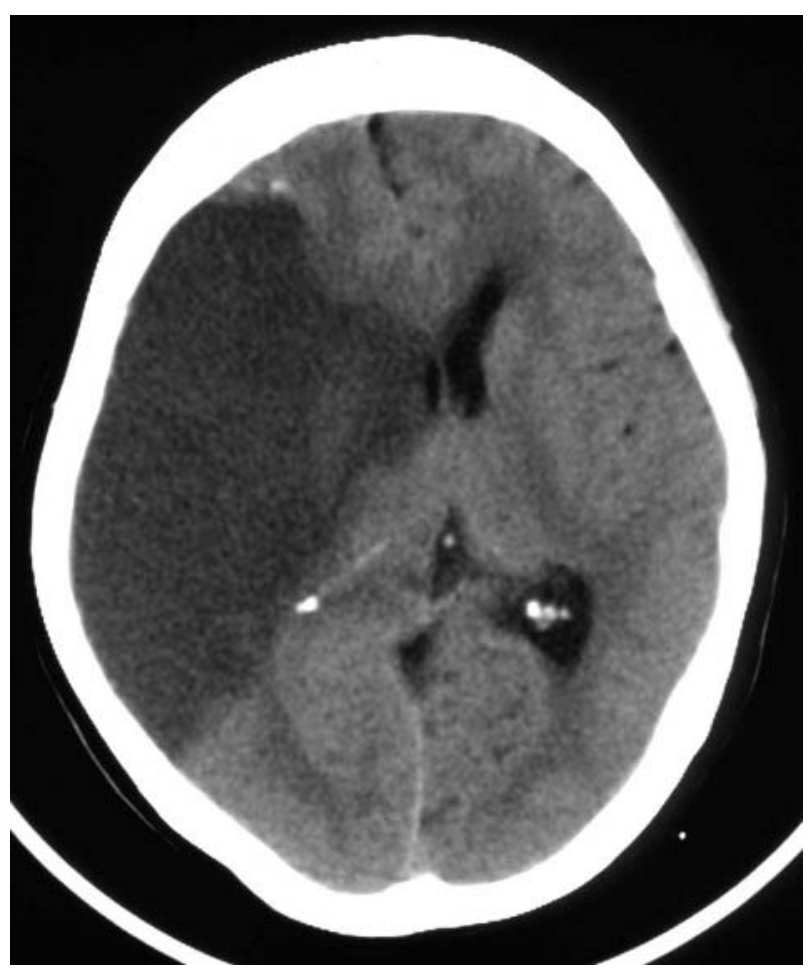

Figure 2 Computed tomography brain scan showing attenuation of the corticomedullary differentiation in right middle cerebral artery territory.

\section{Statistical analysis}

The scoring of the CT image, presence or absence of individual radiological features, age distribution, existence of risk factors, and the NIHSS score on admission were correlated with the 30 day mortality. Fisher's exact test was used to evaluate $2 \times 2$ time tables. Wilcoxon rank test was used to compare the age distribution. The presence of individual risk factor was evaluated by a single explanatory variable search and factors with $\mathrm{p}<0.05$ were entered into stepwise logistic regression analysis and factors with $\mathrm{p}<0.05$ in the logistic regression analysis were considered as independently predictive factors. All statistical analyses were performed by the SPSS Version 11.0 (Chicago, Illinois, USA) installed in a personal computer.

\section{RESULTS}

The NIHSS on admission ranged from 2-40, with a mean of 20 and a median score of 18 . The overall 30 day mortality of these 55 patients was $25.5 \%$. There was no significant difference in age distribution, frequency of atrial fibrillation, diabetes mellitus, hypertension, ischaemic heart disease, and smoking in survivors and deceased patients (Table 1 ).

Twenty two patients had the examination performed $<6$ hours. Thirty three patients had the examination performed 6-24 hours after stroke onset. The mean time lapsed between the examination and the onset of symptoms was 7.3 hours $+/-5.5$ hours ( $1 \mathrm{SD}$ ).

There was very good correlation between the two radiologists reading the images independently. The third reader was required to resolve disagreement only in two to three occasions in scoring of extent of MCA infarct, hyperdense MCA sign, effacement of subarachnoid space and cella media, and attenuation of corticomedullary differentiation. The interobserver kappa values for scoring of these CT features were $0.93,0.96,0.94$, and 0.97 , respectively. Single explanatory variable analysis showed NIHSS, presence of midline shift, midline shift of more than $1 \mathrm{~cm}$, extent of 
Table 1 Characteristics of survivors and non-survivors

\begin{tabular}{|c|c|c|c|}
\hline \multicolumn{2}{|l|}{ Characteristics } & \multirow{2}{*}{$\begin{array}{l}\text { Survivors } \\
18\end{array}$} & \multirow{2}{*}{$\begin{array}{l}\text { Non-survivors } \\
6\end{array}$} \\
\hline Gender & Male & & \\
\hline & Female & 23 & 8 \\
\hline Age (mean $+/-1$ & D, years) & $74.8+1-9.9$ & $78.6+/-7.5$ \\
\hline $\begin{array}{l}\text { NIHSS on admissi } \\
\text { (mean }+/-1 \text { SD) }\end{array}$ & & $16.8+/-10.5$ & $31.1+/-8.5$ \\
\hline \multirow[t]{2}{*}{ Atrial fibrillation } & Present & 11 & 8 \\
\hline & Absent & 30 & 6 \\
\hline \multirow[t]{2}{*}{ Diabetes mellitus } & Present & 10 & 1 \\
\hline & Absent & 31 & 13 \\
\hline \multirow[t]{2}{*}{ Hypertension } & Present & 28 & 7 \\
\hline & Absent & 13 & 7 \\
\hline \multirow{4}{*}{$\begin{array}{l}\text { Ischaemic heart } \\
\text { disease } \\
\text { Smoking }\end{array}$} & Present & 9 & 2 \\
\hline & Absent & 32 & 12 \\
\hline & Present & 13 & 2 \\
\hline & Absent & 28 & 12 \\
\hline \multirow[t]{2}{*}{ Hyperlipidaemia } & Present & 14 & 4 \\
\hline & Absent & 27 & 10 \\
\hline
\end{tabular}

infarct, presence of hydrocephalus, the effacement of subarachnoid space, attenuation of corticomedullary differentiation, and the total CT features scoring were associated with the 30 day mortality. ROC was constructed to obtain the total CT features scoring and NIHSS for the optimum prognostic value. The sensitivity, specificity, positive predictive value, and negative predictive value and their 95\% confidence interval of these CT features and NIHSS were shown in Table 2.

Logistic regression analysis showed the extent of infarct $(p<0.01)$ and NIHSS $(p<0.05)$ were significant parameters in the prediction of 30 day mortality.

When $>33 \%$ of MCA territory involvement was used to predict mortality, it gave a sensitivity of $100 \%$, specificity of $56 \%$, positive predictive value of $44 \%$, and negative predictive value of $100 \%$. When $>67 \%$ of MCA territory involvement was used for the prediction, a sensitivity of $93 \%$, specificity of $95 \%$, positive predictive value of $87 \%$, and negative predictive value of $98 \%$ were achieved.

When both NIHSS $>22$ and $>33 \%$ of MCA involvement was used to predict survival, it gave a sensitivity of $86 \%(95 \%$ confidence interval of $67-100 \%$ ), specificity of $98 \%$ (93$100 \%)$, positive predictive value of $92 \%(78-100 \%)$, negative predictive value of $95 \%$ (89-100\%), and an accuracy of $94.5 \%$. However, when both NIHSS $>22$ and $>67 \%$ of MCA involvement was used to predict survival, it gave a sensitivity of $100 \%$ (95\% confidence interval of $100-100 \%$ ), a specificity of $86 \%$ (75-96\%), positive predictive value of $68 \%$ (48-89\%), negative predictive value of $100 \%(100-100 \%)$, and an accuracy of $89.1 \%$.

\section{DISCUSSION}

CT of the brain is commonly performed for patient with suspected stroke. It is easily accessible and the subminute scanning time allows assessment of patients who are not cooperative or unstable clinically. Despite the higher sensitivity of detection of acute stroke by magnetic resonance imaging, CT still plays a significant role in assessment of acute stroke.

The 30 day mortality of extensive middle cerebral artery is up to $25.5 \%(14 / 55)$ in our study. The mortality is considerably higher than that of $15 \%$ reported by $\mathrm{Ng}$ and Mimmannitya. ${ }^{2}$ This might be related to the high incidence of malignant MCA infarction $(14.5 \%, 8 / 55)$ in our group. Malignant MCA infarct is defined as extensive MCA infarct with a midline shift. Malignant MCA infarct carries a high mortality, up to $80 \%$, even with maximum conservative intensive care. ${ }^{3}$ The poor prognosis is associated with secondary haemorrhage, involvement of respiratory centre
Table 2 Computed tomography features for prediction of mortality

\begin{tabular}{llllll}
\hline & NIHSS $>22$ & & Extent $>\mathbf{6 7 \%}$ & & TS $>\mathbf{5}$ \\
\cline { 2 - 3 } Prevalence & $\mathbf{2 5}$ & & $\mathbf{1 5}$ & & $\mathbf{1 0}$ \\
\hline Sensitivity (\%) & $86(67-100)$ & & $100(100-100)$ & & $71(48-95)$ \\
Specificity (\%) & $98(93-100)$ & & $86(75-96)$ & & $100(100-100)$ \\
PPV (\%) & $92(78-100)$ & & $68(48-89)$ & & $100(100-100)$ \\
NPV (\%) & $95(89-100)$ & & $100(100-100)$ & $91(83-99)$ \\
\hline
\end{tabular}

NIHSS, National Institutes of Health Stroke Scale score; NPV, negative predictive value; PPV, positive predictive value; TS, total computed tomography features scoring.

$95 \%$ confidence interval was given in parenthesis.

in brain stem stroke and transtentorial herniation. ${ }^{3}$ Maximal brain swelling occurs 3-5 days after stroke ${ }^{5}{ }^{6}$ and herniation is the most frequent cause of death.

In view of the poor prognosis of these patients, decompressive hemicraniectomy might offer a beneficial therapeutic option. ${ }^{7}$ Early identification of patients with high mortality and offer of surgical treatment to suitable candidates might reduce mortality. Only very few studies have evaluated whether radiological features demonstrated by CT have any prognostic value. ${ }^{89}$ Other studies have evaluated the predictive value of $\mathrm{CT}$ features in the development of malignant MCA infarct, which signifies high mortality and poor prognosis. ${ }^{10}$ In our study, we showed that there was a high interobserver kappa value for scoring of the CT features, indicating the repeatability and reliability of the CT features read by an experienced neuroradiologist/ neurologist.

In Haring's study, ${ }^{10}$ all patients underwent CT scanning within 18 hours after stroke onset. Compression of cella media shows high sensitivity $(80.6 \%)$, specificity $(74.2 \%)$, and positive predictive value $(75.8 \%)$. Hemispheric brain swelling and early midline shift yield a high specificity (96.7$100 \%)$ and positive prediction value $(85.7-100 \%)$ but very low sensitivity (12.9-19.4\%). Attenuated corticomedullary contrast is found to exhibit high specificity (up to 96.8\%) and sensitivity (up to $87.1 \%$ ). Their study has emphasised on the prediction of development of malignant MCA infarct, which carries a high mortality. ${ }^{10}$ In von Kummer et al's series, ${ }^{8}$ MCA territory hypodensity $>50 \%$ ( sensitivity of $61 \%$, specificity of $98 \%$, and positive predictive value of $85 \%$ ) and local brain swelling (sensitivity of $78 \%$, specificity of $83 \%$, and positive predictive value of $70 \%$ ) are found to be good predictors of mortality. Our study has analysed CT features predicting mortality in extensive MCA infarct. Similar diagnostic imaging criteria for the prediction of survival and the prediction of development of malignant MCA infarct, which was associated with high mortality, were adopted by other authors. ${ }^{8}{ }^{10-13}$

Our results are closely associated with previous findings. The presence of risk factors and age are not good indicators of the survival. This might be related to the small size of population in the study and the results might not be representative of all acute stroke patients. These patients might also be a distinct group and the presence of the extensive MCA infarct might outweigh the predictive value of age and other vascular risk factors. Clinically, NIHSS on admission is the most useful predictor of outcome. A NIHSS $>22$ is predictive of high mortality. Radiologically, demonstration of a midline shift, extent of midline shift, presence of obstructive hydrocephalus, or hyperdense MCA is specific for poor prognosis. However, brain oedema is maximised in 2472 hours $^{14}$ and these signs demonstrated by an early CT examination are not sensitive enough to predict the final 
outcome. The development of early oedema (effacement of subarachnoid space, cella media, the attenuation of corticomedullary differentiation) and the infarct extent give high sensitivity, specificity, positive and negative predictive value for the outcome. As malignant MCA infarction carries a high mortality, it would not be surprising for radiological features to carry a significant predictive value for both development of malignant MCA infarct and mortality, such as attenuation of corticomedullary differentiation.

Presence of hyperdense MCA sign signifies the MCA trunk occlusion and is found to be related to a fatal outcome. ${ }^{10} 1516$ Our results agree with the previous findings; however, the sensitivity is not sensitive enough for prediction of the outcome of individual patient.

Pullicino $e t a l^{17}$ find a strong positive correlation between the extent of midline shift and the risk of early death. Barber et $a l^{9}$ also found that anteroseptal shift of equal to or more than $5 \mathrm{~mm}$ and infarction beyond the MCA territory were significant predictors for fatality - it should be noted that in those 201 patients recruited in their study, CT was performed within 48 hours of stroke onset. In our analysis of CT performed within 24 hours, association between degree of midline shift and mortality can also be established; however, because of delayed development of brain oedema, it is a highly specific sign but not of sufficient sensitivity.

Extent of infarct is an independent prognostic factor in survival, though its predictive value in development of malignant MCA infarct is less significant. When $>67 \%$ of MCA territory involvement was used for the prediction, the specificity was high. Obviously when an extent of $>33 \%$ was chosen for the prediction of mortality, the sensitivity would increase and the specificity would decrease. The choice of a more sensitive or a more specific predictive factor might also be modified by the availability and cost effectiveness of the management of the patients. The development of malignant MCA infarct is more dependent on the mass effect asserted. Ample potential space available to accommodate the swollen brain might preclude the development of malignant MCA infarct. Whether different predictive value of radiological features should affect selection of candidates for decompressive craniectomy remains open.

In summary, although a number of CT features were associated with early mortality, the extent of infarct was the only independent radiological predictive identified and with the NIHSS the attending clinicians would be able to predict early death.

There are two main weaknesses in our study. Both weaknesses are related to the relative small population size. Our analysis does not take the delay between stroke onset and the development of CT abnormality into account. If the patient population is large enough, it will be desirable to assess the predictive value of each CT feature in relation to different timing after onset of clinical symptoms - for example, <3 hours, in 3-6 hours, 6-24 hours, 24-48 hours. Moreover, we have studied a total of 19 CT predictive factors. There is a low event per variable ratio, which might produce over fitting results. Assuming a mortality of $25 \%$ in this group of patients, 760 patients have to be recruited to achieve an event per variable ratio of 10 . Both weaknesses of the study could only be overcome by a large scale multicentre trial.

\section{CONCLUSION}

Survival in extensive MCA infarcts are associated with early CT features demonstrating mass effect (the presence and degree of midline shift, obstructive hydrocephalus, effacement of subarachnoid space/cella media). Extent of infarct and attenuation of corticomedullary differentiation are the two most sensitive and specific single explanatory variables for prediction of survival. By logistic regression analysis, NIHSS and extent of infarct are the only two independent predictors for 30 day survival.

\section{Authors' affiliations}

W W M Lam, W C W Chu, D T K Yeung, Department of Diagnostic

Radiology and Organ Imaging, The Chinese University of Hong Kong, Hong Kong

T W H Leung, L K S Wong, Department of Medicine and Therapeutics, The Chinese University of Hong Kong, Hong Kong

W S Poon, Department of Surgery, The Chinese University of Hong Kong, Hong Kong

Competing interests: none declared

\section{REFERENCES}

1 Saur D, Kuchinski T, Grzyska U, et al. Sensitivity and interrater agreement of $C T$ and diffusion weighted MR imaging in hyperacute stroke. AJNR Am J Neurol 2003;24:878-85

$2 \mathrm{Ng}$ LKY, Mimmannitya J. Massive cerebral infarction with severe brain swelling: a clinicopathological study. Stroke 1970;1:158-63.

3 Hacke W, Schwab S, Horn M, et al. Malignant middle cerebral artery territory infarction: clinical course and prognostic signs. Arch Neurol 1996;53:309-15

4 Berrouschot J, Sterker M, Bettin S, et al. Mortality of space occupying (malignant) middle cerebral artery infarction under conservative intensive care. Intensive Care Med 1998;24:620-3.

5 Shaw CM, Alvord EC, Berry GR. Swelling of the brain following ischemic infarction with arterial occlusion. Arch Neurol 1959;1:161-77.

6 Berry GR, Alpers BJ. Occlusion of the carotid circulation: pathologic considerations. Neurology 1957;7:223-37.

7 Walz B, Zimmermann C, Bottger S, et al. Prognosis of patients after hemicraniectomy in malignant middlle cerebral artery infarction. J Neurol 2002;249: 1183-90.

8 Von Kummer R, Meyding-Lamade U, Forsting M, et al. Sensitivity and prognostic value of early computed tomography in middle cerebral artery trunk occlusion. AJNR Am J Neuroradiol 1994;15:9-15.

9 Barber PA, Demchuk AM, Zhang J, et al. Computed tomographic parameters predicting fatal outcome in large middle cerebral artery infarction. Cerebrovas Dis 2003;16(3):230-5.

10 Haring HP, Dilitz E, Allua A, et al. Attenuated corticomedullary contrast: an early cerebral computed tomography sign indicating malignant middle cerebral artery infarction. Stroke 1999;30:1076-82.

11 Wall SD, Brant-Zawadzki M, Jeffrey RB, et al. High frequency CT findings within 24 hours after cerebral infarction. AJR Am J Roentgenol 1982;138:307-11.

12 Tomura N, Uemura K, Inugami A, et al. Early CT findings in cerebral infarction. Radiology 1988;168:463-7.

13 Truwit CL, Barkovich AJ, Gean-Marton A, et al. Loss of the insular ribbon: another early CT sign of acute middle cerebral artery infarction. Radiology 1990;176:801-6.

14 Haring HP, Aichner FT. Treatment of intracranial hypertension in acute ischemic stroke. In: Castillo J, Dàvalos A, Toni D, eds. Management of Acute Ischemic Stroke. Barcelona, Spain: Springer-Verlag, 1997:109-26.

15 Hacke W, Kaste M, Fieschi C, et al for the ECASS study group. Intravenous thrombolysis with recombinant tissue plasmingogen activator for acute hemispheric stroke: the European Cooperative acute stroke study (ECASS). JAMA 1995;274:1017-25.

16 Launes J, Ketonen L. Dense middle cerebral artery sign: an indicator of poor outcome in middle cerebral artery infarction. J Neurol Neurosurg Psychiatry 1987;50:1550-2.

17 Pullicino PM, Alexandrov AV, Shelton JA, et al. Mass effect and death from severe acute stroke. Neurology 1997;49:1090-5. 